\title{
Blast simulator testing of structures: Methodology and validation
}

\author{
T. Rodriguez-Nikl ${ }^{\mathrm{a}, *}$, G.A. Hegemier ${ }^{\mathrm{b}}$ and F. Seible ${ }^{\mathrm{b}}$ \\ ${ }^{a}$ Oregon State University, School of Civil \& Construction Engineering, Corvallis, OR, USA \\ ${ }^{\mathrm{b}}$ University of California, San Diego, Department of Structural Engineering, Jacobs School of Engineering, San \\ Diego, CA, USA
}

Received 4 August 2008

Revised 2010

\begin{abstract}
The blast simulator at the University of California, San Diego is a unique tool for conducting full-scale testing of blast effects on structures without the use of explosive materials. This blast simulator uses high speed hydraulic actuators to launch specially designed modules toward the specimen, thereby imparting impulse in a blast-like manner. This method of testing offers numerous advantages over field tests with actual explosives, including cost, turn-around time, repeatability, and a clear view of the progression of damage in the specimen. The viability of this method is established by comparing results obtained in the blast simulator with results obtained with actual explosives. The process by which the impulse is imparted to the specimen is then described by a detailed model based on the equivalent single degree of freedom method. Impulse calculated by the model is found to be in good agreement with the experimentally recorded values. Calculated impulse is found to be relatively insensitive to assumptions made about the specimen's resistance function (often not well known before a test) implying that the model can be used with confidence in designing an experimental study.
\end{abstract}

Keywords: Blast simulator, blast hardening, blast mitigation, infrastructure protection, single degree of freedom method

\section{Introduction}

Critical infrastructure has proven vulnerable to attack from improvised explosives such as car or truck bombs. Such attacks involve loss of both life and property not only from primary blast effects but also from progressive collapse of the structure, which occurs when localized damage near the source of the blast causes failure in a member that provides critical support to other parts of the targeted structure. This causes the adjoining structure to fail as the damage propagates through the structure in a chain reaction. Thus, although the initial blast may only affect a localized area of the target, the subsequent progressive collapse may increase damage and loss of life many fold. Preventing progressive collapse is of utmost importance and involves, among other mitigation efforts, developing, validating, and deploying new hardening technologies for retrofit and new construction.

Before deploying any new hardening technology it must be validated through tests and predictive computer modeling with high-fidelity, physics-based computational tools. Our aim is to support this mission by developing a new approach to testing that supports both the development of new hardening technologies and calibration data needed to improve the computational models. To accomplish this, we have developed a system that performs blast load simulations on structural components and assemblages in a laboratory environment without the use of explosive materials. The blast simulator at the University of California, San Diego (UCSD blast simulator) offers several advantages over field testing; these include cost, turnaround time between tests, safety, and repeatability. In

\footnotetext{
*Corresponding author. Tel.: +1 541737 2057; Fax: +1 541737 3052; E-mail: rodrigto@engr.orst.edu.
} 


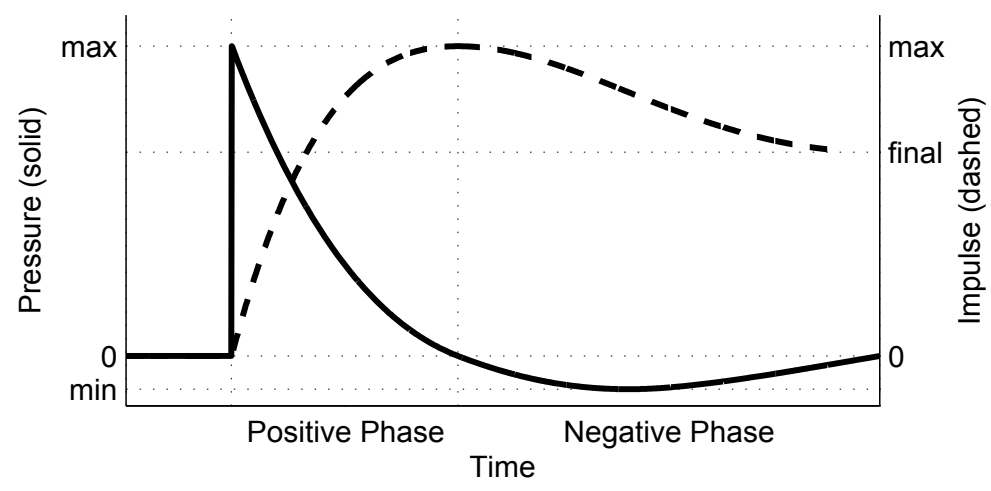

Fig. 1. Typical blast pressure and impulse.

particular, one salient disadvantage of field tests is that the specimen is blocked from view by the fireball produced by the explosive charge; in contrast, we can capture the entire evolution of damage with high resolution, high speed video, which yields much richer information about the mechanisms involved in failure. As will be discussed further, the UCSD blast simulator can accurately replace field testing as long as (1) the characteristic response time of the specimen is much longer than the energy deposition time of the explosive, and (2) the pressure time history of the explosive has a negligible negative phase. This scenario is representative of loading applied to critical structural members (whose failure could lead to progressive collapse) by large, close-in charges.

The UCSD blast simulator adds to existing blast simulation facilities and methods. In the 1960s, in response to the Limited Test Ban Treaty, the High Explosive Simulation Technique was developed to simulate the effect of nuclear explosives by underground detonation of high explosives [22]. Since then there have been numerous test using conventional explosives to simulate the effects of nuclear blasts [14,16]. Currently in the United States, there are shock tube facilities in operation in Vicksburg, Mississippi (the Blast Load Simulator run by the US Army Engineer Research and Development Center) [17] and in White Sands, New Mexico (the Large Blast/Thermal Simulator run by the Defense Threat Reduction Agency) [15]. Similar facilities exist in other countries [21]. Shock tubes use a controlled detonation or sudden release of compressed gas at one end of a highly reinforced tube to apply a load to a specimen at the other [16]. Also in operation are impact-based devices such as those at Florida State University [3]. This kind of device uses gravity to accelerate a single mass toward the specimen and can impart impact and blast-like loads.

An explosive imparts load to a structure through a shock wave of increased air pressure that travels away from the explosive. This shock wave can be amplified as is reflects off a solid surface. The load imparted by an explosive is characterized by the pressure and the specific impulse time histories (Fig. 1). Specific impulse, referred to simply as "impulse," is the time integral of pressure. Pressure rises almost instantaneously from zero to the peak, decays exponentially, drops below zero, and then returns to zero once more. The impulse rises quickly at first, peaks as the pressure vanishes, and then drops during the negative phase. Large, close-in charges such as a car or truck bomb at curbside have durations between $2 \mathrm{~ms}$ and $4 \mathrm{~ms}$, negligible negative phases, and impulse maxima roughly from $11 \mathrm{MPa} \cdot \mathrm{ms}$ to $15 \mathrm{MPa} \cdot \mathrm{ms}$ (1600 psi-ms to $2200 \mathrm{psi} \cdot \mathrm{ms})[1,21,26]$. As long as the characteristic period of the specimen is at least four times greater than the pulse duration, the impulse dominates the specimen response and the exact shape of the pressure pulse is irrelevant [4]. Most structural elements fall into this period range. In these cases we can accurately test the element in the UCSD blast simulator by accurately matching the total impulse; accurately matching the pressure time history is not necessary. Thus, although the blast does not apply load by increasing air pressure, but rather by transferring momentum from a large mass to the specimen, the specimen will respond in the same way under actual airblast as under the loading imparted in the UCSD blast simulator.

A brief overview of the UCSD blast simulator is useful at this time. The most important component of the blast simulator is an array of blast generators supported by a strong wall (Fig. 2). The specimen is supported by a second strong wall and the two are connected by a strong floor. As used in this blast simulator, a blast generator (BG) is a high speed hydraulic actuator with close-coupled, high pressure, nitrogen accumulators (Fig. 3) that can quickly bring a large mass to high speed. It is this mass that, upon impact with the specimen, imparts the required impulse 


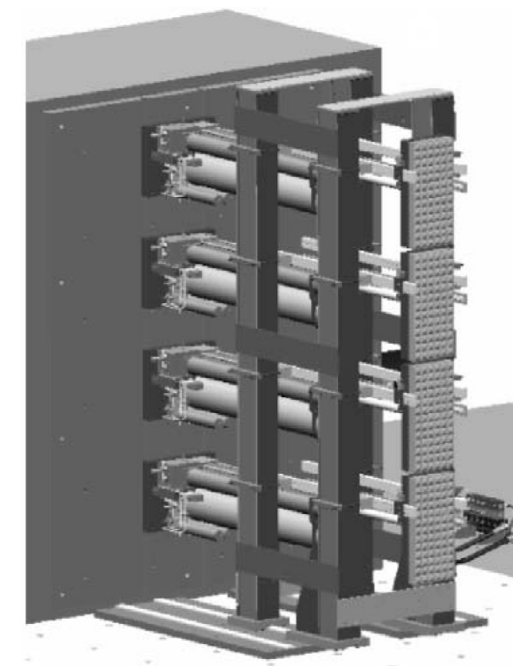

Fig. 2. Array of blast generators with impacting modules attached [20].

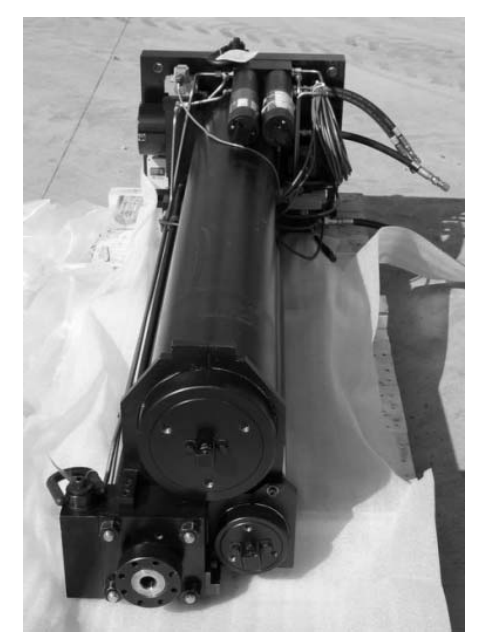

Fig. 3. Blast generator [20].

to the specimen. In contact with the specimen during impact is a specially-formulated pad whose material and geometric properties are designed to give a pressure pulse of the desired duration and shape (Fig. 4). This pad is called the "programmer" and assembled together with the mass (e.g., a steel or aluminum block) is referred to as the "impacting module" or "impactor." The block is stiff enough to spread the load over the front face of the specimen and the programmer modulates the forces of the impact to prevent overly-abrupt, impact-like forces. This is accomplished though a combination of the programmer's viscoelastic material properties and the geometry of the front surface, which is shaped like shallow pyramids, making the programmer gain stiffness gradually as the pyramids compress. Precision valves in the BGs are programmed to open and close according to the desired blast profile. With the tools developed in this paper we can determine the blast generator (BG) settings required to apply a desired impulse. Each BG can be operated separately, giving us the ability to simulate realistic scenarios such as a large, close-in charge with different peak pressures and arrival times at different parts of the column. Detailed system specifications can be found elsewhere $[6,8,18]$.

Once the UCSD blast simulator imparts the desired impulse, the specimen responds as it would under a real explosion. The structural response is measured with displacement gauges, accelerometers, strain gauges, and high speed video with point tracking software. Using the equivalent uniform load method presented herein, one can 


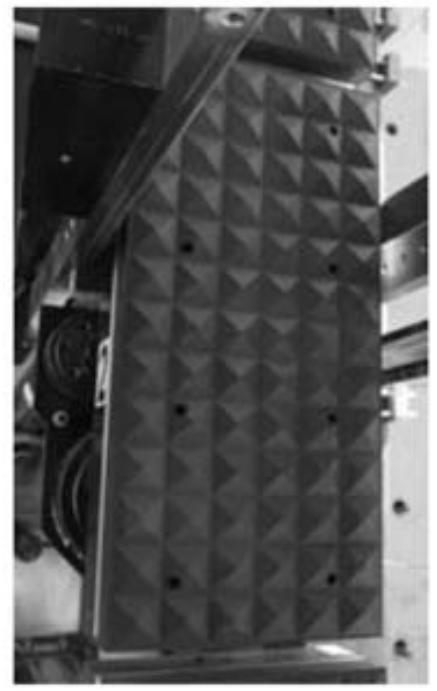

Fig. 4. Programmer. The pyramidal texture on the front surface is designed prevent an overly-abrupt pulse initiation [6].

determine the charge size and standoff that correspond to the observed structural response. Details of structural response are beyond the scope of this paper; preliminary results from ongoing studies of reinforced concrete (RC) building columns, unreinforced masonry and RC walls, steel building columns, and steel bridge towers can be found elsewhere $[6,8]$.

In the following section we introduce a newly developed method of data analysis called the equivalent uniform load method. This method is used throughout the rest of the paper. Next, because the UCSD blast simulator uses a new method of testing, before discussing it in detail, we present data from a series of column tests to demonstrate how UCSD blast simulator results compare to field results with actual explosives. Next is a detailed, mathematical description of the system. The mathematical model, built on equivalent single degree of freedom principles, is then validated against independent test data. Following this we show that first and foremost, the model is a good predictor of the impulse imparted to the specimen. We also show that the model is insensitive to assumptions made about the specimen's resistance, which is often not well known before the test. Lastly, we find that the model is in reasonable agreement with the shape of the pressure pulse applied to the specimen.

\section{Equivalent uniform load method}

Typically, the specimen is impacted by more than one BG. The velocity and time of impact of each BG can vary both because of test design and random error. Making meaningful comparisons between tests requires treating these differences in a rational way. The equivalent uniform load (EUL) method, based on the equivalent single degree of freedom (SDOF) method, was developed to this end [18].

In the equivalent SDOF method a structural element of mass density $m(x)$, length $L$, nonlinear internal resistance $r_{n l}(x, t)$, and subject to a time-varying distributed load $w(x, t)$ is represented by a single degree of freedom system whose properties are based on an assumed deflected shape $\psi(x)$, typically the deflected shape under statically-applied uniform load ( $x$ represents the longitudinal axis of the element and $t$ represents time). To obtain the equivalent SDOF system, the displacement $v$ is first decomposed into spatial and temporal components as $v(x, t)=\psi(x) z(t)$; $\psi(x)$ is usually normalized to range between 0 and 1 , in which case, and if the specimen is symmetric about its midspan, $z(t)$ is equal to the magnitude of midspan deflection. Next, using virtual work, the original (infinite degree of freedom) governing differential equation is converted to the following equivalent single degree of freedom differential equation: $\tilde{M} \ddot{z}(t)+\tilde{r}(t)=\tilde{f}(t)$, where $\tilde{M}=\int_{L} m(x) \psi^{2}(x) \mathrm{d} x$ is the equivalent single degree of freedom (SDOF) mass, $\tilde{r}(t)=\int_{L} r_{n l}(x, t) \psi(x) \mathrm{d} x$ is the equivalent single degree of freedom (SDOF) restoring force, and $\tilde{f}(t)=\int_{L} w(x, t) \psi(x) \mathrm{d} x$ is the equivalent applied single degree of freedom (SDOF) force. Damping 
has been ignored because it does not significantly affect the peak response. Additional details on the SDOF method can be found in most textbooks on structural dynamics [1,4]. Details on the resistance function $r_{n l}$ can be found in blast design manuals [23-26]. More sophisticated extensions to the SDOF method can be found elsewhere in the literature [10-13].

The goal of the EUL method is to compute a uniform pressure $\bar{p}(t)$ that produces the same SDOF force $\tilde{f}(t)$ as the experimentally recorded pressure $p(x, t)$, which varies in both time and space. The recorded pressure is the sum of the individual pressures applied by the $N$ BGs, i.e., $p(x, t)=\sum_{n=1}^{N} p_{n}(x, t)$. The function $p_{n}(x, t)$ is defined mathematically over the whole length of the column. Because each BG can only apply a force where it is in contact with the column, $p_{n}(x, t)$ is equal to zero everywhere except between the BG's bottom and top endpoints, $x_{b_{n}}$ and $x_{t_{n}}$ respectively. The equation governing the equivalence is

$$
\int_{L} p(x, t) \cdot \psi(x) \mathrm{d} x=\int_{L} \bar{p}(x) \cdot \psi(x) \mathrm{d} x .
$$

This yields the equivalent uniform load

$$
\bar{p}(t)=\frac{1}{\mathcal{C}_{f}} \sum_{n=1}^{N} p_{n}(t) \cdot \mathcal{C}_{f_{n}},
$$

where

$$
\mathcal{C}_{f}=\int_{L} \psi(x) \mathrm{d} x \quad \text { and } \quad \mathcal{C}_{f_{n}}=\int_{x_{b_{n}}}^{x_{t_{n}}} \psi(x) \mathrm{d} x .
$$

In essence, this procedure is a weighted sum of the individual effects of each BG, with more weight given to those that are more influential in exciting the assumed mode of displacement.

In the UCSD blast simulator the applied pressure is measured indirectly by multiplying the experimentally recorded acceleration by the mass of the impacting module and dividing by its area. This is acceptable in most cases because the hydraulic forces applied by the BG are usually negligible compared to the force applied to the specimen. With the EUL calculated in this manner, the equivalent impulse can easily be related back to the corresponding charge size and standoff by using standard design charts such as those in TM 5-1300 [26].

\section{Comparison between blast simulator and field test data}

A parallel study on reinforced concrete (RC) columns has been conducted in both the blast simulator and in field tests using actual explosives. We now compare numerical and qualitative results from both cases to show that they are equivalent. RC columns have been field tested by Karagozian and Case (K\&C) both in test buildings and as separate specimens in a special test rig [2]. The columns were mid-sized, square columns with widely spaced shear reinforcement as is typical in non-seismic design. Some specimens were unretrofitted (as-built) while others were retrofitted with carbon fiber fiber reinforced polymer (CFRP) jackets: some with fibers in the hoop direction to provide shear strength and confinement, and others, subsequently referred to as a carbon shell system (CSS), with hoop reinforcement and additional fibers in the longitudinal direction to increase flexural strength. Identical columns have been tested in the UCSD blast simulator $[7,18]$. In total there are seven field tests and ten laboratory tests that can be compared. In these tests, impulse demands ranged from $6.8 \mathrm{MPa} \cdot \mathrm{ms}$ to $16.9 \mathrm{MPa} \cdot \mathrm{ms}(990 \mathrm{psi} \cdot \mathrm{ms}$ to $2450 \mathrm{psi} \cdot \mathrm{ms}$ ). When compared, both types of tests are consistent in the observed impulse time histories, failure modes and demand-damage curves.

\subsection{Impulse time histories}

As is observed in Fig. 5, the shapes of impulse time histories from the field and blast simulator at the University of California, San Diego (UCSD blast simulator) are equivalent by engineering standards. This figure shows typical time histories normalized by dividing by their respective maxima and with the time scales shifted so the curves match at $10 \%$ of the maximum. It is clear that the two shapes are very similar. They differ significantly only in the slow initiation of impulse in laboratory tests. This is by design and is caused by the pyramidal shape on the front of the programmer. 


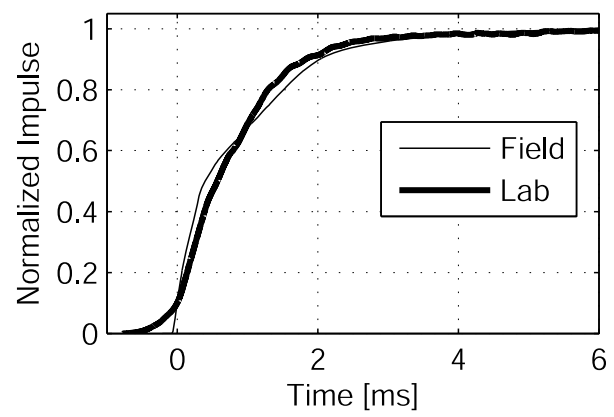

Fig. 5. Comparison of typical impulse time history shapes.
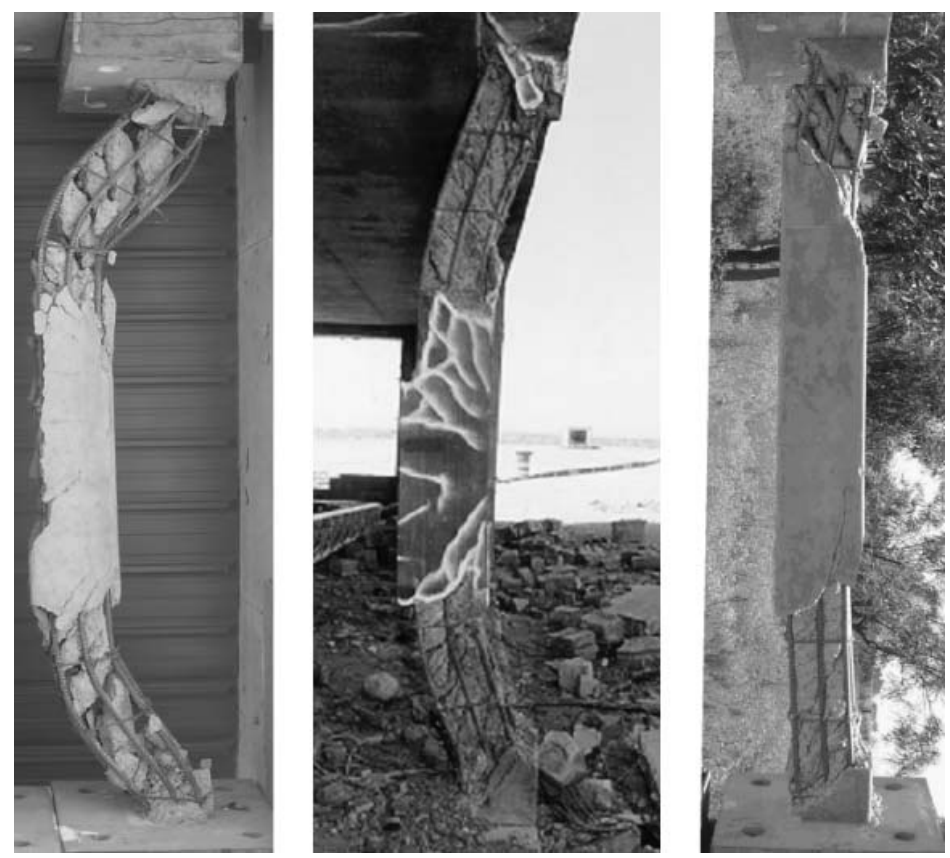

Fig. 6. As-built columns tested in the field (center) and in the laboratory (left and right) at different impulse levels (increasing from right to left) [18].

\subsection{Failure modes}

In Fig. 6 are as-built columns tested in the UCSD blast simulator and in the field at various impulse levels. They all exhibit the same failure mode: shear failure at the top and bottom of the column and a relatively undamaged center portion. With increasing impulse demand we observe, as expected, increased residual deflection, increased length of the zone of shear failure, and increased degradation of the central section. In Fig. 7 are two carbon shell system (CSS) columns tested at a load level equivalent to a car bomb at curbside; one was tested in the field and the other in the UCSD blast simulator. We observe that both columns are practically undamaged.

\subsection{Demand-damage curves}

To make the comparisons more systematic, we consider demand-damage relations for each specimen type. Demand is defined as the impulse applied to the specimen. Blast simulator tests use impulse as obtained by the EUL method. For the field tests, whose instrumentation was not reliable enough to make the same calculation, we take 


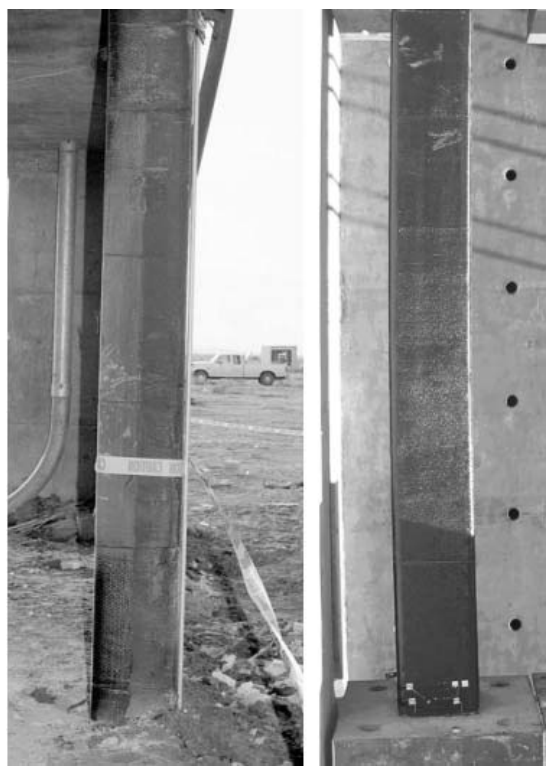

Fig. 7. CSS columns tested in the field (left) and in the laboratory (right), both at impulse levels corresponding to a car bomb at curbside [18].

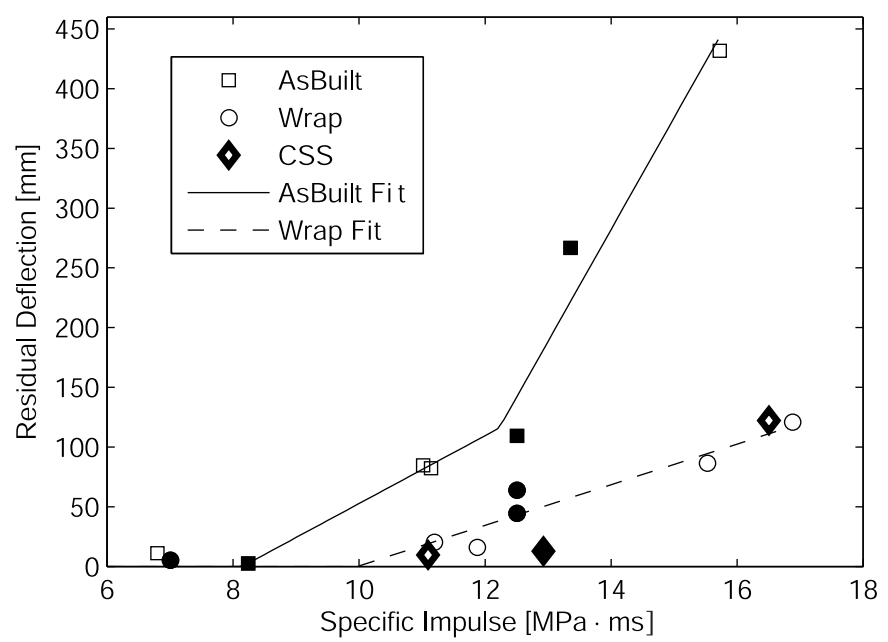

Fig. 8. Demand-damage data for column tests. Field tests are indicated with filled symbols and laboratory tests with hollow symbols.

the theoretical value of specific impulse as calculated by the CONWEP computer program [9]. The damage metric is the residual midheight deflection.

We expect that at low demand there will be no damage. As demand increases, damage will increase, but this damage will not be severe. However, with further increases in demand, the damage will become catastrophic and after a certain point will increase quickly with little additional demand. We also expect that stronger specimens will remain undamaged at higher demand and will reach catastrophic damage at higher demand levels. Thus the demand-damage curve for the wrapped specimens should lie below that for the as-built specimens, and the curve for the CSS specimens should lie lower still.

Indeed, this is the trend found in the experimental data (Fig. 8). In addition to the data points, Fig. 8 shows piecewise linear, least squares fits to the as-built and wrapped column data; these are our best estimates of the demand damage curves. The as-built columns, denoted with the square marker, see incipient damage at about $8 \mathrm{MPa} \cdot \mathrm{ms}$, 
and the rate of damage increases just past $12 \mathrm{MPa} \cdot \mathrm{ms}$. Both the laboratory and field data are tightly clustered about the fitted curve with a coefficient of determination $\left(R^{2}\right.$ value) of 0.976 . The wrapped columns, denoted by a circular marker, remain undamaged at higher demands than the as-built columns and for every level of demand they suffer lower damage. These columns never experience catastrophic damage. The data are also well clustered about the fitted curve with an $R^{2}$ value of 0.925 . Lastly, we observe the CSS data, denoted with a diamond marker. There are not enough points to make a meaningful curve fit, so we look only at individual data points. Consistent with expectations, the CSS specimens remain undamaged longer than the wrapped columns. The only damaged CSS specimen had damage somewhat higher than expectations, but taken as a whole these data make a strong argument that results from the UCSD blast simulator are equivalent to field tests using actual explosives.

\section{Detailed system description}

Having demonstrated the validity of UCSD blast simulator results vis-à-vis field testing, we now describe from a theoretical point of view the blast-like load produced by the UCSD blast simulator during a laboratory test. This description, built upon the SDOF method, has been incorporated into a computer program for use at the blast simulator facilities [19]. There are three parts to the model: the kinetics of the impacting modules as they approach and impact the specimen, the kinetics of the specimen as it is impacted, and the equal and opposite programmer force acting on each of them. Each part is discussed individually.

\subsection{Kinetics of the impacting module}

The kinetics of the impacting modules are complicated. They involve the dynamics of the BGs, e.g., hydraulic flows, oil pressures, gas compression, servo-controlled valve behavior, and dissipative forces. They also include modeling of the connection between the impacting module and the BG. However, much of this information is proprietary and cannot be discussed in detail. Thus, we simply describe the kinetics of the $n^{\text {th }}$ impacting module as

$$
\ddot{v}_{n}(t)=\frac{F_{b g, n}(t)-F_{p, n}(t)}{M_{n}},
$$

where $\ddot{v}_{n}(t)$ is the acceleration of the $n^{\text {th }}$ impacting module, $M_{n}$ its mass, $F_{b g, n}(t)$ the BG forces acting on it, and $F_{p, n}(t)$ the programmer force. In most cases, the BG forces during impact are negligible with respect to the programmer forces. Thus, if it were necessary, these forces could usually be neglected with little error. BG forces are, however, vitally important in determining the time and velocity of impact, which can be predicted to within \pm $1.2 \%$ on average and $\pm 4.7 \%$ in the worst case [19].

\subsection{Kinetics of the specimen}

The specimen's behavior is described by the SDOF method as follows:

$$
\ddot{z}(t)=\frac{\tilde{f}(t)-\tilde{r}(t)}{\tilde{M}} .
$$

These terms have been defined in Section 2 . The resistance $\tilde{r}(t)$ is typically not written directly as a function of time, but parametrically as a function of $z(t)$ and, if nonlinear, $\dot{z}(t)$ to account for load reversals. With an applied distributed load varying in both time and space, the applied SDOF force is

$$
\tilde{f}(t)=\int_{L} w(x, t) \cdot \psi(x) \mathrm{d} x .
$$

In the blast simulator, the applied load is the sum of the loads at each of the $N$ impacting modules. Each module is centered at $x=X_{n}$ and has a length $h_{n}=x_{t_{n}}-x_{b_{n}}$. We can then define the applied load as

$$
w(x, t)=\sum_{n=1}^{N} w_{n}(x, t),
$$


where

$$
w_{n}(x, t)=\left\{\begin{array}{cc}
F_{p, n}(t) / h_{n}, 2\left|x-X_{n}\right| \leqslant h_{n} \\
0, & \text { otherwise. }
\end{array}\right.
$$

The programmer force $F_{p, n}(t)$ will be defined shortly. We have assumed that the force developed by the programmer is uniform. This is an approximation as it has been shown that due to confinement effects, the force is greater at the center than the edges [19].

\subsection{Programmer force}

The programmer is a nonlinear, viscoelastic material, thus the force developed by the programmer is a function of the deformation $d_{n}(t)$ and deformation rate $\dot{d}_{n}(t)$. We use separation of variables to describe the force in the programmer as follows:

$$
F_{p, n}(t)=F_{p, n}\left(d_{n}(t), \dot{d}_{n}(t)\right)=f_{s, n}\left(d_{n}(t)\right) \cdot f_{d, n}\left(\dot{d}_{n}(t)\right)
$$

The static portion carries the subscript $s$ while the dynamic modification carries the subscript $d$. We define the programmer deformation and deformation rate by the motion of a representative reference point. The reference deformation of the $n^{\text {th }}$ programmer is equal to the displacement of the impacting module, $v_{n}(t)$, minus the displacement of the specimen at $x=X_{n}$. Thus

$$
d_{n}(t)=v_{n}(t)-\psi\left(X_{n}\right) z(t)
$$

Differentiating, we obtain the deformation rate:

$$
\dot{d}_{n}(t)=\dot{v}_{n}(t)-\psi\left(X_{n}\right) \dot{z}(t)
$$

If $d$ becomes negative this is interpreted to mean that the impacting module is not in contact with the specimen. In this case $\dot{d}$ is read as the rate at which the impacting module is approaching or receding from the specimen (relative to the specimen).

The static curve is based on previous experimental work that showed that the programmer follows a power law, namely

$$
f_{s, n}(t)=\mathrm{S}_{n} \beta_{1}\left(d_{n}(t)\right)^{\beta_{2}} .
$$

$\beta_{1}$ and $\beta_{2}$ have been fitted experimentally to a reference programmer, and $S_{n}$ is a shape coefficient that extends the curve to arbitrary geometry [19]. The shape coefficient uses the concept of a shape factor $s$, defined as the area of one loaded face divided by the unloaded area. A square prism made of a material of Young's modulus $E$ has an apparent modulus equal to $E_{a}=E\left(1.00+2.20 s^{2}\right)$ [5]. Applying this concept we obtain

$$
\mathrm{S}_{n}=\frac{A_{n}\left(1.00+2.20 s_{n}^{2}\right)}{A_{r}\left(1.00+2.20 s_{r}^{2}\right)},
$$

where $A$ refers to area, subscript $r$ to the reference programmer, and subscript $n$ to the $n^{\text {th }}$ programmer. Figure 9 shows the measured and calculated reference curves. For this curve, if $d$ is measured in millimeters, $\beta_{1}=28.36 \mathrm{kN}$ and $\beta_{2}=1.993$ with an $R^{2}$ value of 0.99 [18].

To capture the appropriate physical behavior, the dynamic modification factor $f_{d, n}$ should be equal to unity when the deformation rate is zero, it should increase when the deformation rate is positive, and decrease when deformation rate is negative. It should behave symmetrically about unity as it increases or decreases, except that because the contact condition cannot develop tension, the dynamic modification factor may not be negative. In terms of the nondimensionalized parameter $\gamma_{n}(t)=\dot{d}_{n}(t) /(30 \mathrm{~m} / \mathrm{s})$, we propose that the dynamic modification can be represented by a third order polynomial:

$$
f_{d, n}\left(\gamma_{n}(t)\right)=1+\operatorname{sign}\left(\gamma_{n}(t)\right)\left[\beta_{3}\left|\gamma_{n}(t)\right|+\beta_{4} \gamma_{n}^{2}(t)+\beta_{5}\left|\gamma_{n}^{3}(t)\right|\right],
$$

where $\beta_{3}, \beta_{4}$, and $\beta_{5}$ are fitting parameters and $\operatorname{sign}(x)$ equals 1 if $x>0,-1$ if $x<0$, and 0 otherwise.

To fit the three parameters we use ballistic impact test data obtained with the UCSD blast simulator. In these tests a mass was fired at various velocities toward a target of similar mass. The incoming and outgoing velocities were 


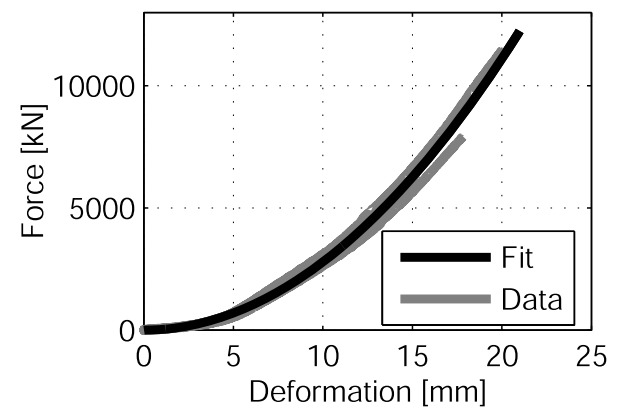

Fig. 9. Loading curves used to fit parameters $\beta_{1}$ and $\beta_{2}$ along with least squares fit [18].

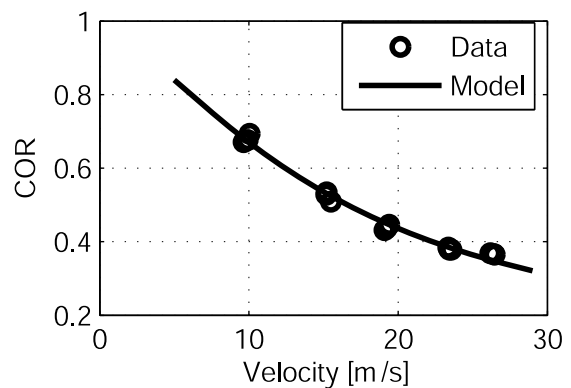

Fig. 10. COR data points used to fit parameters $\beta_{3}$ to $\beta_{5}$ along with least squares fit [19].

measured and the coefficient of restitution (COR) was calculated (COR is equal to the relative speed of separation after impact divided by the relative speed of approach before impact). This yielded an experimental COR-versusincoming-velocity curve. Each test was then modeled by the above described process and the COR was calculated (a shape factor modification was used because the programmer in these tests was a different shape than the one used to obtain the static reference curve). This yielded a theoretical COR-versus-incoming-velocity curve. The parameters were adjusted until the sum square error between the theoretical and experimental curves was minimized. Figure 4.3 shows the experimental and converged theoretical COR-versus-incoming-velocity curves on one of the programmers. The dynamic modification factor obtained by this fit is shown in Fig. 11. In this case, $\beta_{3}=1.1817$, $\beta_{4}=4.2403$, and $\beta_{5}=3.6168$ with a coefficient of determination ( $R^{2}$ value) better than 0.98 [19].

The parameters $\beta_{1}$ to $\beta_{5}$ that have been given above are for the programmer that was used in the column tests. However, it should be realized that the particular values are of little importance when describing the model in general terms. Any programmer can be described by this model provided there are static load-deflection curves and ballistic impact test data for calibration. Once the parameters are determined for the programmer in question, the force developed by the programmer is given by substituting Eqs (7), (8), and (9) into Eq. (6). This force acts on the impacting modules according to Eq. (1) and on the specimen according to Eqs (2), (3), (4), and (5). This is a highly coupled problem that must be solved numerically.

\section{Validation}

The model from the previous section has been implemented in an explicit time stepping algorithm that has been checked for convergence as well as for agreement with theoretical results. After these preliminary checks it was validated against independently obtained test data [19]. This process yielded two important conclusions. First, impulse, the most important determinant of load on the specimen, is well predicted with little scatter. Second, for specimens whose natural period is sufficiently small as compared to the pulse duration, the prediction of impulse is insensitive to the specimen resistance. This is important because it ensures that an estimate of load will be accurate even when the expected specimen behavior is not well known, and it allows us to use the same BG settings for each blast scenario irrespective of the specimen being tested. Furthermore, the model also gives useful information about the pulse shape. 
Table 1

Maximum deviation from the mean of each response parameter as the resistance function is changed (as a percentage of the mean)

\begin{tabular}{cccc}
\hline Impulse & Peak Pressure & Time of Peak & Pulse Time \\
\hline $4.5 \%$ & $0.22 \%$ & $0.33 \%$ & $10.2 \%$ \\
\hline
\end{tabular}

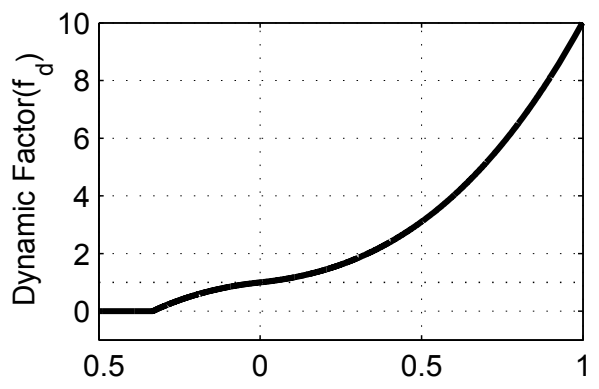

Fig. 11. Dynamic factor resulting from least squares fit [19].

\subsection{Low sensitivity to resistance function}

The sensitivity study was conducted on the six wrapped column specimens discussed above. For each test the experimentally recorded velocity and time of the impact were input to the model and the process was modeled up to the ultimate specimen displacement. The specimen was modeled with four different resistance functions for each test. The weakest resistance function was calculated as per the DAHS Manual [23], which considers elasto-plastic flexural hinge behavior and arch action. The strongest resistance function assumed purely elastic behavior. Between these two extremes were two hypothetical resistance functions. The as-built specimens were not used in this study because they fail in shear at low deflections when differences in resistance functions are negligible.

In addition to studying the effect of the resistance function on impulse, three additional load parameters were considered: magnitude and time of maximum pressure, and pulse time (defined as the time elapsed from when the pressure first exceeds $10 \%$ of maximum to the time when it last drops below the same limit). These parameters give information about the shape of the pulse. While we are concerned only with specimens whose behavior is not dependent on the shape of the pulse, it is still of interest in evaluating the model to study how closely the calculation resembles the recorded pressure pulse.

The model was run for each scenario under consideration and for each resistance function for a total of twenty-four runs. For each loading parameter we computed the mean of the calculated values and the maximum deviation from the mean value. Impulse differed by no more than $4.5 \%$ as the resistance function was changed. Peak pressure and the time at which it occurs differed by much less while pulse time varied more (Table 1). The time and magnitude of peak pressure are less sensitive than impulse and pulse time because they occur earlier in the response when the specimen has not started to move appreciably and the differences in resistance function have not yet manifested themselves. On the other hand, impulse and pulse time depend on the entire duration of response; as such they are more sensitive to changes in resistance function.

These results indicate that the proposed model is relatively insensitive to choice of resistance function and can be used to estimate the load applied to a test before the behavior of the specimen is well known. As a word of caution, this conclusion only applies to specimens whose period is at most one quarter of the pulse duration. In other cases, a large part of the specimen response will take place during the pulse and the interaction between the impacting modules and the specimen will be greater. Additional data would be needed to assess the model under these circumstances.

\subsection{Agreement with test data}

The model was compared against all available data for column tests obtained in the UCSD blast simulator. We observe in Fig. 12 that the pressure time histories are similar in the experiment and the calculation (this figure and 


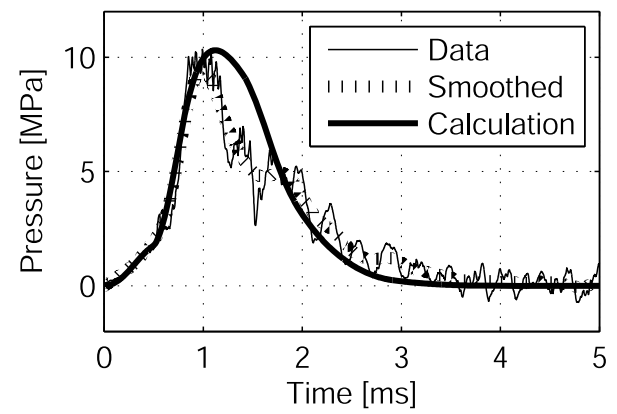

Fig. 12. Example of calculated versus recorded pressure.

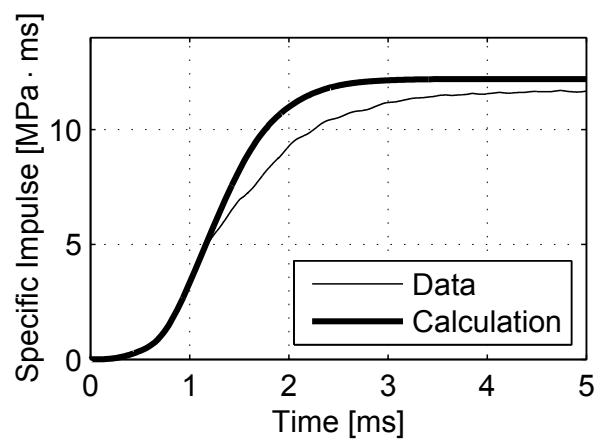

Fig. 13. Example of calculated versus recorded impulse.

the next were chosen to be indicative of typical results and as such exhibit neither the best nor the worst agreement). Initially the calculated and recorded pressures are close. Near the peak, the recorded pressure usually drops below the calculation. Later in the record it is usually higher than the calculation. These differences are thought to be from shock waves traveling through the system, e.g., in the oil in the blast generator (BG) or in the impacting mass (recall that the pressures are obtained indirectly via the accelerations). The model does not account for these higher order effects. However, the acceleration is recorded on the rear face of the impacting module and it is thought that the accelerations resulting from shock waves are significantly filtered by the programmer before they arrive at the specimen. Turning now to the impulse (Fig. 13), we see that when the recorded pressure drops below the calculation the recorded impulse does the same, but once the recorded pressure crosses above the calculation the impulse catches up. The ultimate result is that the calculated impulse is close to the recorded impulse, although the recorded impulse takes longer to reach this maximum value.

The experimental and calculated impulse values for the ten tests are compared in Fig. 14. In addition to a direct comparison of calculation and data, this figure displays the residuals, trendlines, and a $95 \%$ confidence interval. Thus we obtain an idea not only of the mean error, but how accurate we might expect the model to be in the future. The model slightly overestimates the data by an average value of $0.74 \mathrm{MPa} \cdot \mathrm{ms}(110 \mathrm{psi} \cdot \mathrm{ms})$. The discrepancy is somewhat lower at higher levels of impulse. A future prediction can be expected with $95 \%$ certainty to be no more than 2.6 MPa.ms (380 psi-ms) away from the correct value. This is an excellent agreement.

Because the model enforces conservation of momentum and is calibrated against experimental data to properly model energy losses, we expect it to predict impulse with little error. However, there have been no a priori assumptions made about the shape of the pulse. Rather, the pulse is dynamically generated in each simulation as the impacting module interacts with the specimen. Thus we expect a weaker agreement between pulse shape parameters and the data. Indeed, this is what is observed. Magnitude of peak pressure tends to be overpredicted by $1.5 \mathrm{MPa}$ (220 psi) on average, time of peak pressure tends to be underpredicted by $0.13 \mathrm{~ms}$ on average, and pulse time tends to be underpredicted by $0.63 \mathrm{~ms}$ on average. By themselves, these are in very good agreement with the data. However, the agreement becomes weaker when confidence intervals are taken into account. In addition, at higher impacting velocities, time of peak pressure and pulse time become more sensitive to the higher order effects discussed above. 

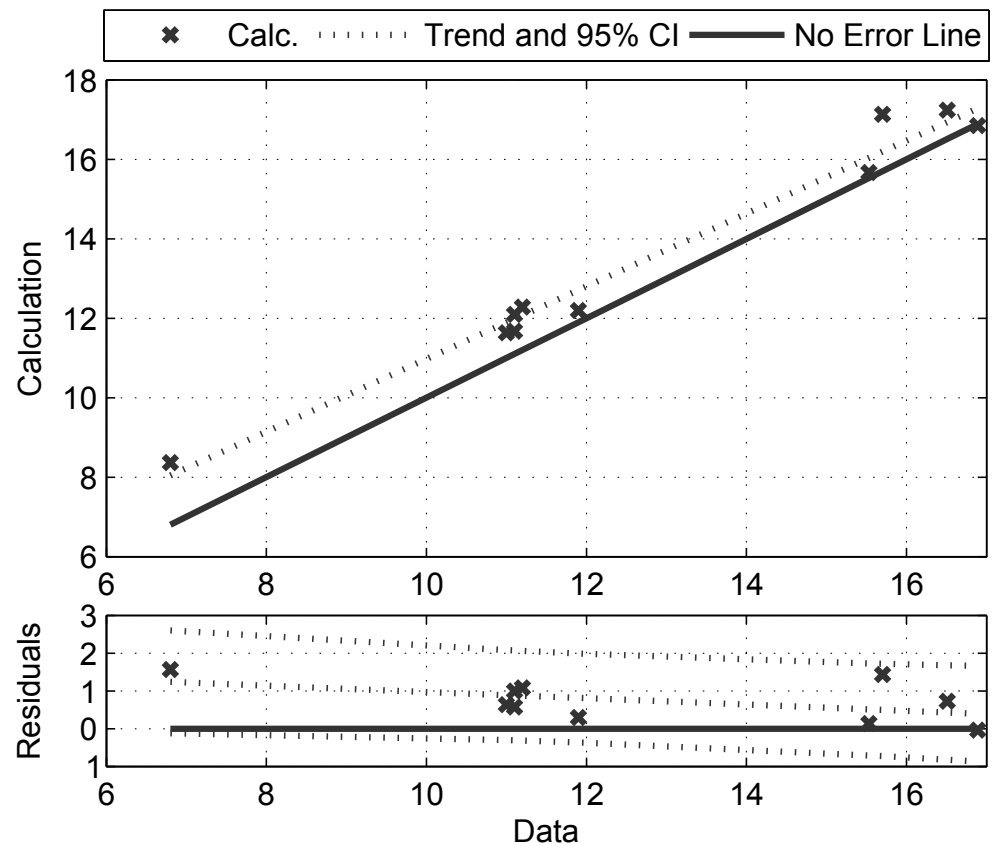

Fig. 14. Calculated impulse compared to data for ten tests (MPa.ms) [19].

In summary, the model performs very well at its intended purpose - to predict the impulse delivered to the specimen. The prediction has been shown to be accurate despite potential uncertainty in the resistance function of the specimen and higher order effects that are not captured by the model. In addition, the model provides estimates of the expected pulse shape, albeit with less certainty than the impulse prediction. As such, it is a useful predictive and analytical tool for laboratory testing in the UCSD blast simulator or similar devices that might be designed in the future.

\section{Conclusion}

The UCSD blast simulator is a unique tool for conducting full-scale testing of blast effects on structures without the use of actual explosives. This method of testing offers numerous advantages over testing with explosive charges including cost, turn-around time, repeatability, and a clear view of the progression of damage in the specimen. In Section 3 we established the viability of the UCSD blast simulator by comparing laboratory results of column tests to similar field tests conducted with actual explosives. The laboratory tests exhibited the same loading, failure modes and demand-damage patterns as field tests. Following this, we described in detail the process by which the load is applied to the specimen. The derived model was based on the equivalent SDOF method and an experimental characterization of blast simulator components. This model was then compared to column test data obtained in the laboratory to validate it and characterize its accuracy. The model was found to predict impulse with little error and little scatter. In addition, the impulse prediction was shown to be insensitive to the resistance function of the specimen and to some higher order effects typically observed in the data. Moreover, the model was shown to predict the shape of the pressure pulse applied to the specimen. This has established the model as a useful tool for designing experiments in the service of improved blast hardening technologies and for the safety of critical infrastructure.

\section{Acknowledgements}

This work was supported by the Technical Support Working Group. The blast generators were developed in cooperation with MTS Systems Corporation. Karagozian and Case and SAIC have provided additional analytical support on blast simulator projects. 


\section{References}

[1] J.M. Biggs, Introduction to Structural Dynamics, McGraw-Hill, 1964.

[2] BLast Information System (BLIS) database, Technical Support Working Group, 2004, BLIS-HD-1.0.0-0018.

[3] Center for Infrastructure Protection and Physical Security, Facilities, <http://www.cipps.eng.ufl.edu/site/facilities/>, 18 Oct 2009.

[4] A.K. Chopra, Dynamics of Structures, Pearson Prentice-Hall, 2007.

[5] A.N. Gent and P.B. Lindley, The compression of bonded rubber blocks, Proc Instn Mech Engrs 173 (1959), 111-122.

[6] G. Hegemier, F. Seible, K. Arnett, T. Rodriguez-Nikl, M. Oesterle, J. Wolfson, M. Gram and A. Clark, The UCSD blast simulator, in: Proceedings of the 77th Shock \& Vibration Symposium, Monterey, CA, USA, 2006.

[7] G. Hegemier, F. Seible, M. Oesterle and T. Rodriguez-Nikl, Explosive Loading Laboratory Testing for Blast Mitigation: Blast Simulator Operations and Steel Cellular Bridge Tower Testing, Quick Look Report 1 CFRP Retrofitted and As-Built RC Columns, UCSD Structural Engineering, La Jolla, CA, USA, 2006.

[8] G.A. Hegemier, F. Seible, T. Rodriguez-Nikl and K. Arnett, Blast mitigation of critical infrastructure components and systems, in: Proceedings of the Second fib Congress, Naples, Italy, 2006.

[9] D. Hyde, ConWep: Conventional weapons effects, U.S. Army Engineer Waterways Experiment Station, 1992, USAEWES / SS-R.

[10] T. Krauthammer, A. Assadi-Lamouki and H.M. Shanaa, Analysis of impulsively loaded reinforced concrete structural elements-I, Theory, Computers \& Structures 48 (1993), 851-60.

[11] T. Krauthammer, A. Assadi-Lamouki and H.M. Shanaa, Analysis of impulsively loaded reinforced concrete structural elements-II, Implementation, Computers \& Structures 48 (1993), 861-871.

[12] T. Krauthammer, N. Bazeos and T. Holmquist, Modified SDOF analysis of RC box-type structures, Journal of Structural Engineering 112 (1986), 726-744.

[13] T. Krauthammer, S. Shahriar and H. Shanaa, Response of reinforced concrete elements to severe impulsive loads, Journal of Structural Engineering 116 (1990), 1061-1079.

[14] National Academy of Sciences, Protecting Buildings from Bomb Damage: Transfer of Blast Mitigation Technologies from Military to Civilian Applications, National Academy Press, 1995.

[15] National Academy of Sciences, Blast Mitigation for Structures: 1999 Status Report on the DTRA/TSWG Program, National Academy Press, 2000

[16] Office of the Deputy Assistant to the Secretary of Defense for Nuclear Matters, Nuclear matters: A practical guide, 2008.

[17] S.D. Robert and C.F. Johnson, Blast response of conventional and high performance reinforced concrete panels, in: Structures 2009, American Society of Civil Engineers, 2009.

[18] T. Rodriguez-Nikl, Experimental Simulations of Explosive Loading on Structural Components: Reinforced Concrete Columns with Advanced Composite Jackets, Ph.D. dissertation, University of California, San Diego, 2006.

[19] T. Rodriguez-Nikl, M. Gram, M.G. Oesterle, G.A. Hegemier and F. Seible, The UCSD Blast Simulator: Experimental Methodology, Research Report SSRP-2007/05, UCSD Structural Engineering, La Jolla, CA, USA, 2008.

[20] T. Rodriguez-Nikl, C.-S. Lee, G.A. Hegemier and F. Seible, The UCSD Blast Simulator: Design and Construction, Research Report SSRP-2007/04, UCSD Structural Engineering, La Jolla, CA, USA, 2008.

[21] P.D. Smith and J.G. Hetherington, Blast and Ballistic Loading of Structures, Butterworth-Heinemann, 1994.

[22] H.L. Taylor, Design and Construction of Test Facilities to Simulate the Effects of a Nuclear Detonation: HANDEC I and HANDEC II, Technical Report AFWL-TR-69-171, Air Force Weapons Laboratory, 1970.

[23] U.S. Defense Special Weapons Agency and Departments of the Army, the Navy and the Air Force, Design and Analyis of Hardened Structures to Conventional Weapons Effects, 1998, DAHSCWEMAN-97 / TM 5-855-1/ NAVFAC P-1080 / AFPAM 32-1147(I).

[24] U.S. Department of Defense, Design and Analyis of Hardened Structures to Conventional Weapons Effects, 2002, uFC 3-340-01. Limited Availability.

[25] U.S. Department of the Army, Fundamentals of Protective Design for Conventional Weapons, 1986, TM 5-855-1.

[26] U.S. Departments of the Army, the Navy and the Air Force, Structures to Resist the Effects of Accidental Explosions (TM 5-1300 / NAVFAC P-397/ AFR 88-22, Revision 1), 1990. 

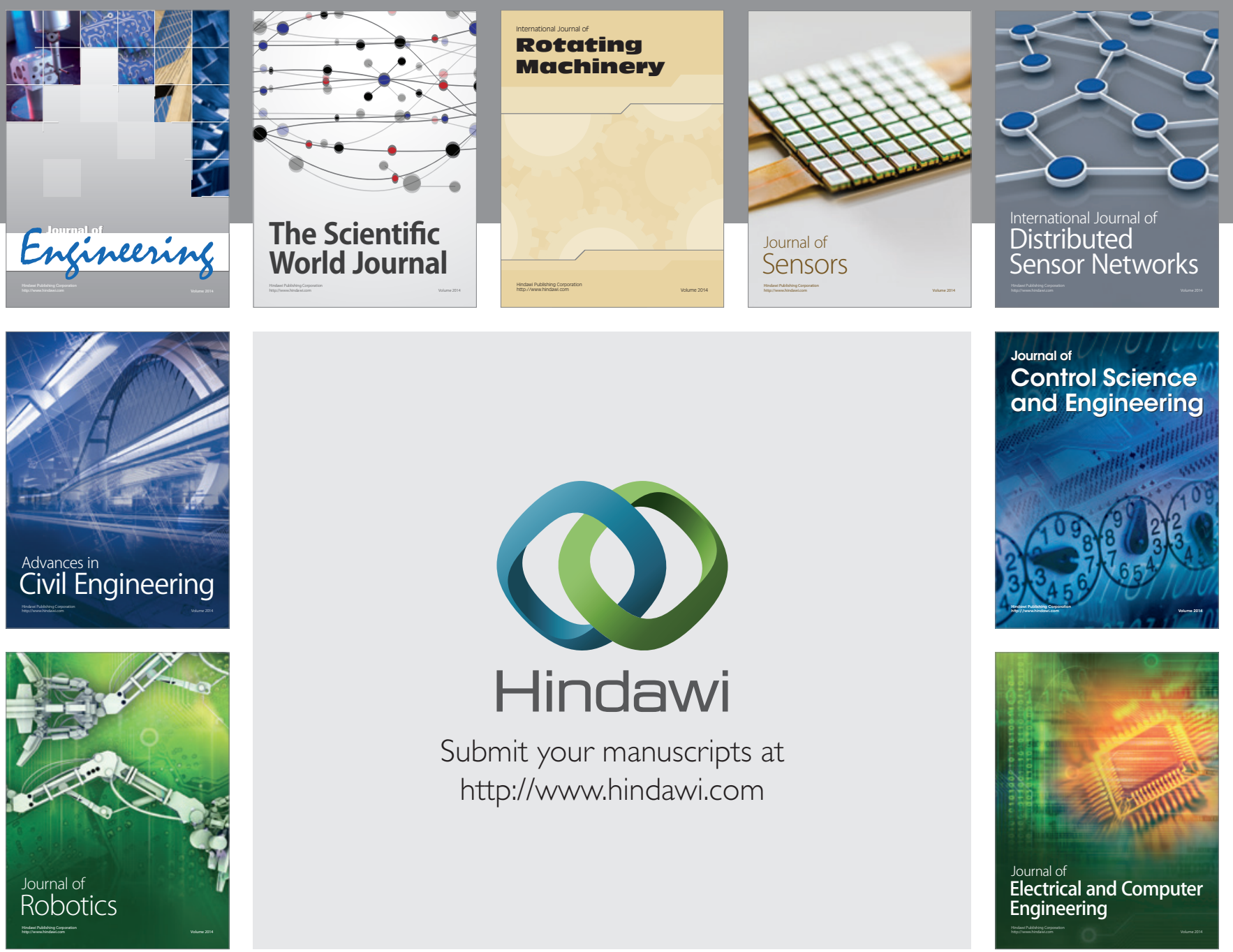

Submit your manuscripts at

http://www.hindawi.com
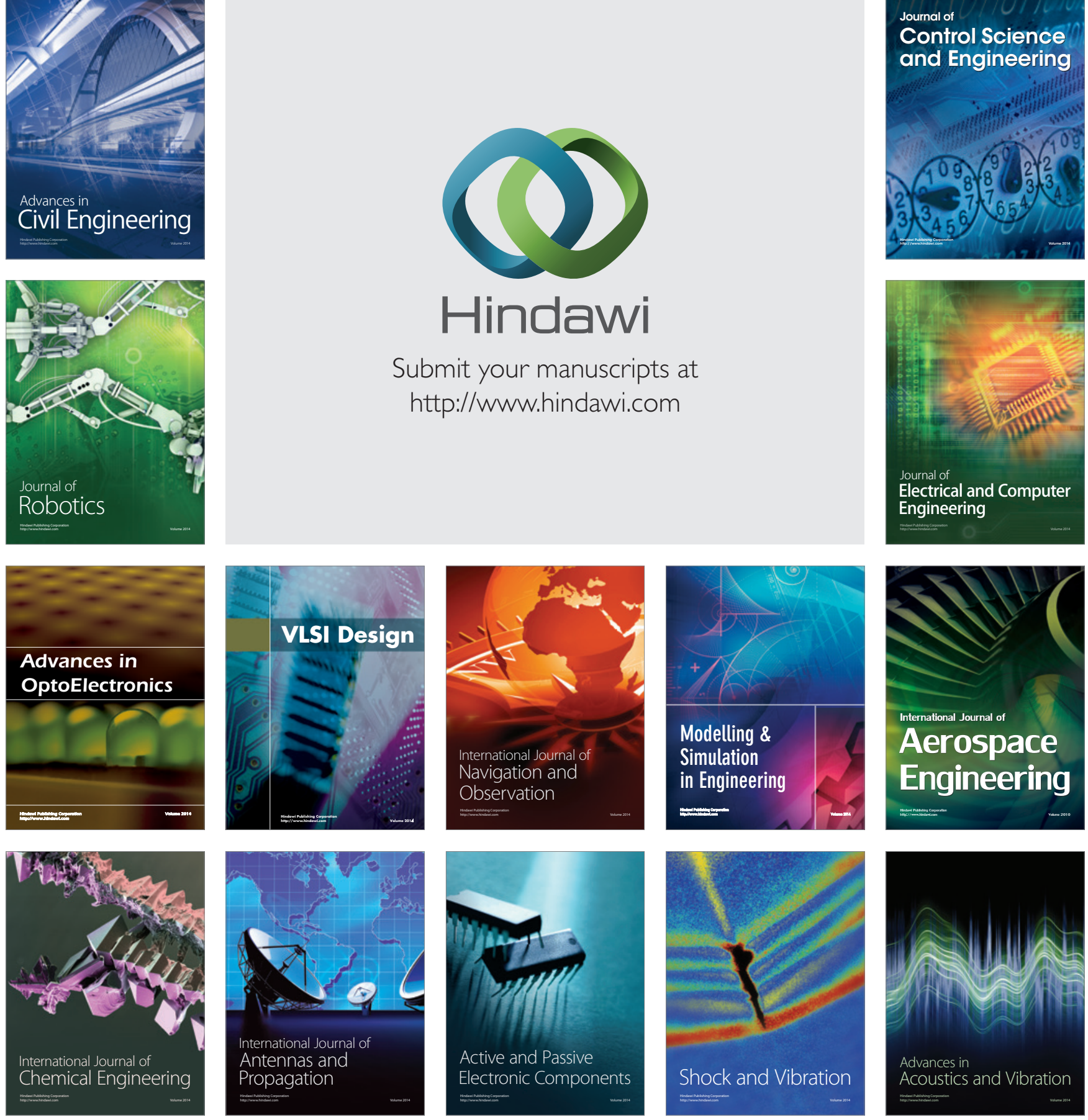